\title{
Article
}

\section{Raman spectroscopic techniques to detect ovarian cancer biomarkers in blood plasma}

Paraskevaidi, Maria, Ashton, Katherine M, Stringfellow, Helen F, Wood, Nicholas J, Keating, Patrick J, Rowbottom, Anthony W, Martin-Hirsch, Pierre L and Martin, Francis L

Available at http://clok.uclan.ac.uk/23949/

Paraskevaidi, Maria, Ashton, Katherine M, Stringfellow, Helen F, Wood, Nicholas J, Keating, Patrick J, Rowbottom, Anthony W, Martin-Hirsch, Pierre L and Martin, Francis L ORCID: 0000-0001-8562-4944 (2018) Raman spectroscopic techniques to detect ovarian cancer biomarkers in blood plasma. Talanta, 189 . pp. 281-288. ISSN 0039-9140

It is advisable to refer to the publisher's version if you intend to cite from the work. http://dx.doi.org/10.1016/j.talanta.2018.06.084

For more information about UCLan's research in this area go to

http://www.uclan.ac.uk/researchgroups/ and search for <name of research Group>.

For information about Research generally at UCLan please go to http://www.uclan.ac.uk/research/

All outputs in CLoK are protected by Intellectual Property Rights law, including Copyright law. Copyright, IPR and Moral Rights for the works on this site are retained by the individual authors and/or other copyright owners. Terms and conditions for use of this material are defined in the policies page.

\section{CLoK}

Central Lancashire online Knowledge www.clok.uclan.ac.uk

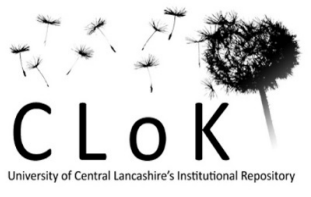




\section{Raman spectroscopic techniques to detect ovarian cancer biomarkers in blood plasma}

Maria Paraskevaidi ${ }^{\mathrm{a}, 1}$, Katherine M. Ashton ${ }^{\mathrm{b}}$, Helen F. Stringfellow ${ }^{\mathrm{b}}$, Nicholas Wood ${ }^{\mathrm{c}}$,

Patrick Keating ${ }^{\mathrm{c}}$, Anthony Rowbottom ${ }^{\mathrm{d}}$, Pierre L. Martin-Hirsch ${ }^{\mathrm{c}}$ and Francis L. Martin ${ }^{\mathrm{a}, 1}$

${ }^{a}$ School of Pharmacy and Biomedical Sciences, University of Central Lancashire, Preston PRI

2HE, $U K$

${ }^{b}$ Pathology Department, Lancashire Teaching Hospitals NHS Foundation Trust, Preston PR2

9HT, $U K$

${ }^{c}$ Department of Obstetrics and Gynaecology, Lancashire Teaching Hospitals NHS Foundation

Trust, Preston PR2 9HT, UK

${ }^{d}$ Immunology Laboratory, Pathology Department, Lancashire Teaching Hospitals NHS Foundation Trust, Preston PR2 9HT, UK

$3{ }^{1}$ To whom correspondence should be addressed. Email: mparaskevaidi@uclan.ac.uk or 24 flmartin@uclan.ac.uk Tel: +44 (0) 1772896482 
Robust diagnosis of ovarian cancer is crucial to improve patient outcomes. The lack of a single and accurate diagnostic approach necessitates the advent of novel methods in the field. In the present study, two spectroscopic techniques, Raman and surface-enhanced Raman spectroscopy (SERS) using silver nanoparticles, have been employed to identify signatures linked to cancer in blood. Blood plasma samples were collected from 27 patients with ovarian cancer and 28 with benign gynecological conditions, the majority of which had a prolapse. Early ovarian cancer cases were also included in the cohort $(n=17)$. The derived information was processed to account for differences between cancerous and healthy individuals and a support vector machine (SVM) algorithm was applied for classification. A subgroup analysis using CA-125 levels was also conducted to rule out that the observed segregation was due to CA-125 differences between patients and controls. Both techniques provided satisfactory diagnostic accuracy for the detection of ovarian cancer, with spontaneous Raman achieving $94 \%$ sensitivity and $96 \%$ specificity and SERS $87 \%$ sensitivity and $89 \%$ specificity. For early ovarian cancer, Raman achieved sensitivity and specificity of $93 \%$ and $97 \%$, respectively, while SERS had $80 \%$ sensitivity and $94 \%$ specificity. Five spectral biomarkers were detected by both techniques and could be utilised as a panel of markers indicating carcinogenesis. CA125 levels did not seem to undermine the high classification accuracies. This minimally invasive test may provide an alternative diagnostic and screening tool for ovarian cancer that is superior to other established blood-based biomarkers.

Keywords: Ovarian cancer; diagnostics; biospectroscopy; Raman; SERS 


\section{Introduction}

Ovarian cancer is frequently discovered at a late stage due to non-specific symptomatology. More than $70 \%$ of ovarian cancer patients are diagnosed at an advanced state (stage IV) when the five-year survival rate is $25 \%$ [1]. Ideally, disease should be diagnosed promptly and at an early stage (stage I), when cancer is completely confined to the ovaries, as stages II, III and IV are considered advanced with cancer being spread outside the ovaries into the pelvis (e.g., fallopian tubes, bladder or rectum), abdominal cavity (e.g., lining of the abdomen or lymph nodes) and other distinct organs (e.g., lungs), respectively [2]. As a consequence, the five-year survival rate of stage II patients is increased to $70 \%$, while for stage I patients it is further increased to $90 \%[1,3]$.

Screening or diagnostic tests for ovarian cancer comprise of cancer antigen CA-125 alone, ultrasound imaging of the ovaries or a combination. These tests have different screening utility depending on whether they are applied in low or high risk populations. However neither, even in combination, have robust levels of diagnostic accuracy $[4,5]$. A variety of blood tests have also been developed with CA 19-9, human epididymis protein 4 (HE4), apolipoprotein A1 (ApoA1), insulin growth factor II (IGF-II) and transferrin being some of them [6-9]. However, most of these individual biomarker tests yield unacceptable diagnostic accuracies which render them unsuitable for clinical use. Recent strategies attempt to combine a number of these tests to achieve superior performance.

Raman spectroscopy has been used extensively in cancer diagnostics utilising a variety of samples (e.g., cells, tissues or biological fluids). Other diagnostic techniques, such as optical coherence tomography, fluorescence microscopy or nonlinear microscopy could also be used for diagnostic purposes, however Raman spectroscopy has been shown in many cases to provide better results [10]. Cervical, skin, breast, oral and brain cancers, as well as other diseases, are some of the wide applications of Raman, facilitating disease detection/monitoring 
74 or even intraoperative assessment of surgical margins [11-19]. Moreover, previous spectroscopic studies have successfully investigated ovarian tissue post-surgery and showed significant differences between normal and malignant cases [20-22]. However, the use of biological fluids, such as blood samples, are numerous: minimally-invasive collection, easier sample preparation and collection of serial samples from the same participants, just to name a few [23, 24]. Raman spectroscopy investigates the phenomenon of inelastic light scattering that is caused after the interaction of light with matter. The sample's electrons first get excited to a virtual state and then fall back to their original energy level either by losing or by gaining energy. The generated shift in energy is characteristic for specific biomolecules such as proteins, lipids and nucleic acids, providing thus invaluable information for a biological sample.

Raman scattering is inherently weak and, therefore, enhancement techniques have been developed to increase the derived signal [25]. Surface-enhanced Raman spectroscopy (SERS) is one of the commonly applied methods which utilises rough metallic surfaces or nanostructures (e.g., silver or gold nanoparticles) to increase the Raman signal by $10^{3}-10^{10}$ times. SERS exploits the great electromagnetic field enhancement that is caused by oscillations of surface electrons, called surface plasmons [26]. This allows detection of molecules at low concentration and can partly account for fluorescence that may distort the spectra [15, 27].

The main objective of this study was to use blood spectroscopy in order to assess the diagnostic accuracy for ovarian cancer in women with both early- and late-stage cancer, which has not been previously attempted. Extraction of differential spectral biomarkers was also performed and tentative assignments were made for the development of a panel of diagnostic markers. An important confounding factor, which has not been taken into account in previous spectroscopic studies, and could lead to falsified classification between cancer and healthy controls was the CA-125 level; therefore its effect on the spectral results has been now 
calculated in a separate subgroup analysis. To the best of our knowledge this is also the first study employing both Raman and SERS to investigate the effect of the enhanced approach in the diagnostic accuracy - does increased signal necessarily imply improved diagnostic accuracy as well?

\section{Materials and Methods}

\section{Population}

This study included 27 women with ovarian cancer (17/27 stage I) and 28 women with benign gynecological conditions or a prolapse. All specimens were collected with ethical approval obtained at Royal Preston Hospital UK (16/EE/0010). Mean-age was 68 years for the cancer group and 56 years for the non-cancer group. More information about the cohort characteristics can be found in Table 1; more detailed information about each participant is given in Table S1 [see Supplementary Information (SI)]. Age difference between the different groups was also taken into account to demonstrate whether it affected the spectral results, and therefore the diagnostic accuracy (see SI Fig. S1). Women who were on Tamoxifen have been excluded.

\section{CA-125 measurement}

CA-125 levels were determined in blood serum samples for both the ovarian cancer patients and healthy individuals. This test, is a two-site sandwich immunoassay using electrochemiluminescence (ECL) technology which uses monoclonal antibodies (Elecsys CA 125 II, Roche Diagnostics GmbH). The system (Roche Cobas 8000) automatically dispenses $20 \mu \mathrm{l}$ of sample into a cuvette and then dispenses a biotinylated CA125-specific antibody and a CA125-specific antibody labelled with ruthenium complex react to form a sandwich complex. Streptavidin microparticles are then added and the complex becomes bound to the solid phase via the interaction of biotin and streptavidin. The reaction mixture is aspirated in to the reaction 
cell where the microparticles are magnetically captured onto the surface of the electrode.

Unbound substances are then removed with Procell solution. Application of a voltage to the electrode induces the chemiluminescent emission, which is measured by the photomultiplier; the results are then determined via a calibration curve. A direct relationship exists between the amount of CA-125 in sample and the amount of photons detected by the system. The reference range of CA-125 is 0-35 units/ml (0-35 kU/L), with values $>35 \mathrm{kU} / \mathrm{L}$ indicating an increased probability for residual or recurrent ovarian cancer in patients treated for primary epithelial ovarian cancer.

\section{Blood plasma preparation for spontaneous Raman and SERS analysis}

Whole blood was collected into EDTA tubes, centrifuged at $2000 \mathrm{rpm}$ for $10 \mathrm{~min}$ to remove the cells (erythrocytes, white blood cells and platelets) from plasma. The supernatant was then collected and stored at $-80^{\circ} \mathrm{C}$ and thawed at room temperature prior to spectroscopic interrogation. After the samples were thawed, $50 \mu \mathrm{L}$ were deposited directly on aluminium foil slides and left to air-dry. In order to employ SERS as an enhancement method, silver nanoparticles (AgNPs), with a diameter of $100 \mathrm{~nm}$, were used (nanoComposix, Inc., San

Diego). The stock solution (mass concentration: $1.02 \mathrm{mg} / \mathrm{ml}$ ) was diluted in phosphate buffered saline (PBS); $1 \mu \mathrm{l} \mathrm{AgNPs} \mathrm{was} \mathrm{diluted} \mathrm{in} 100 \mu \mathrm{l}$ PBS. Fifty $\mu$ l of the diluted solution were mixed with $50 \mu \mathrm{l}$ of the biological fluid and the resulting mixture $(100 \mu \mathrm{l})$ was then deposited on aluminium foil slides and was again left to air-dry at room temperature before Raman spectra were acquired.

\section{Spectral acquisition}

The experimental settings were kept the same for both Raman and SERS analysis. Spectra were collected with an InVia Renishaw Raman spectrometer coupled with a chargecoupled device (CCD) detector and a Leica microscope. A $785 \mathrm{~nm}$ laser was used with a 1200 
run. Seven point spectra were acquired per sample using a 50× objective, 10 second exposure time, 5\% laser power and 2 accumulations in the spectral range of $2000-400 \mathrm{~cm}^{-1}$ to achieve optimum spectral quality.

\section{Spectral pre-processing and classification}

Spectra were evaluated during collection and any cosmic rays were removed by using the Renishaw WiRe software. An in-house developed IRootLab toolbox (http://trevisanj.github.io/irootlab/) was then implemented within MATLAB environment (MathWorks, Natick, USA) for further pre-processing and classification of the data. An initial pre-processing phase is required to deal with any background noise or non-biological variability associated with spectral acquisition or instrumentation. Herein, all spectra were firstly truncated to the biological region $\left(1800-500 \mathrm{~cm}^{-1}\right)$, wavelet denoised, polynomial baseline corrected and vector normalized. All of these steps are standard in the Raman analysis of biological samples in order to generate noise-free spectra with conventional appearance [27]. Difference-between-mean (DBM) spectra was also performed to extract potential biomarkers by subtracting the mean spectra of two classes (i.e., ovarian cancer patients and controls); a peak detection algorithm was implemented to identify the ten most segregating peaks. a classification function from training data. Some of the criteria for the choice of classifier include the achieved diagnostic accuracy, as well as training and computational time [28]. For SVM implementation, the already pre-processed dataset was further normalized (to the $[0,1]$ range) in order to put all the variables on the same scale. We used the Gaussian kernel SVM, which implies that there are two parameters to be tuned to the value that gives best classification: $\mathrm{c}$ and gamma [29]. The optimal tuning parameters were found using grid search (5-fold cross-validation) and then used to calculate the sensitivity and specificity for the 
175 Sensitivity $(\%)=\frac{T P}{T P+F N} \times 100$

$$
\text { Specificity }(\%)=\frac{T N}{T N+F P} \times 100
$$
positive.

\section{Statistical analysis}

\section{Availability of data}

\section{Results} biomolecules.

when the disease is present, while specificity is defined as the probability that a test will be negative at the absence of disease; they were calculated by the following equations:

where TP is defined as true positive; FN as false negative; TN as true negative; and FP as false

The common peaks that were found to differentiate the classes in both Raman and SERS, after the implementation of the DBM algorithm, were further analyzed in GraphPad Prism 7.0 (GraphPad Software Inc., La Jolla, CA, USA). A student's t-test (non-parametric, two-tailed, 95\% confidence interval (CI)) was performed to account for statistical significance with a $P$-value of 0.05 or less being considered significant. Statistical analysis was carried out on averaged spectra in order to account for differences between individuals and not spectra.

All data (raw and pre-processed spectra) along with appropriate code identifiers will be uploaded onto the publicly accessible data repository Figshare.

The enhancement effect of SERS is shown in Fig. 1; after the addition of the AgNPs solution in the blood samples, the Raman signal is notably increased as the silver nanostructures are closely adsorbed to the biomolecules present in the plasma (Fig. 1B). The spectral differences between Raman and SERS spectra were expected and can be attributed to the complex nature of the samples, as well as the nonspecific binding of the nanoparticles to the 
SERS, to calculate the sensitivity and specificity rates with which these methods can distinguish ovarian cancer patients $(n=27)$ and healthy individuals $(n=28)$. For the Raman dataset the achieved sensitivity and specificity were $94 \%$ and $96 \%$, respectively (Fig. 2A); for the SERS dataset sensitivity and specificity were $87 \%$ and $89 \%$, respectively. After the DBM implementation, ten peaks responsible for the differentiation, were selected; out of those, five peaks were picked up by both and Raman and SERS and, therefore, these were used for further statistical analysis (Fig. 3A and 3B). The five peaks that were selected with Raman spectroscopy were: $1657 \mathrm{~cm}^{-1}$ (Amide I, $P=0.0158 ; 95 \% \mathrm{CI}=0.00049$ to 0.00471$), 1418 \mathrm{~cm}^{-}$

${ }^{1}\left(\mathrm{CH}_{2}\right.$ in lipids, $P=0.0034 ; 95 \% \mathrm{CI}=0.00061$ to 0.00334$), 1301 \mathrm{~cm}^{-1}\left(\mathrm{CH}_{2}\right.$ in lipids, $P=$ 206 $0.0612 ; 95 \% \mathrm{CI}=-0.00379$ to 0.00007 ), $1242 \mathrm{~cm}^{-1}$ (Amide III, $P=0.0103 ; 95 \% \mathrm{CI}=-0.00521$ 207 to -0.00066 ) and $916 \mathrm{~cm}^{-1}$ (amino acids/carbohydrates, $P=0.0024 ; 95 \% \mathrm{CI}=0.00111$ to 208 0.0047), while with SERS: $1655 \mathrm{~cm}^{-1}$ (Amide I, $P=0.0351 ; 95 \% \mathrm{CI}=-0.0117$ to -0.0005 ), 209 $1429 \mathrm{~cm}^{-1}\left(\mathrm{CH}_{2}\right.$ in lipids, $P=0.066 ; 95 \% \mathrm{CI}=-0.00049$ to 0.00873$), 1302 \mathrm{~cm}^{-1}\left(\mathrm{CH}_{2}\right.$ in lipids, $P=0.0882 ; 95 \% \mathrm{CI}=-0.00825$ to 0.00079$), 1257 \mathrm{~cm}^{-1}$ (Amide III, $P=0.0003 ; 95 \% \mathrm{CI}=-$ 0.00916 to -0.00283 ) and $919 \mathrm{~cm}^{-1}$ (amino acids/carbohydrates, $P=0.0004 ; 95 \% \mathrm{CI}=-0.0067$ to -0.00163$)$.

In order to show that the achieved accuracy was not just due to the difference in the CA-125 levels between cancer patients and healthy individuals, we also performed the SVM classification after taking into account the different protein levels. Sensitivity and specificity remained exceptionally high: Raman yielded $99 \%$ sensitivity and $85 \%$ specificity after comparing individuals with CA-125>35 (Fig. 4A), as well as 78\% sensitivity and 99\% specificity for individuals with CA-125<35 (Fig. 4B); SERS achieved sensitivity and specificity of $96 \%$ and $74 \%$, respectively, for the group with CA-125>35 (Fig. 4C), as well as $72 \%$ sensitivity and $97 \%$ specificity for the CA-125<35 group (Fig. 4D). Similarly, we 
considered the age difference between the different groups using the spectra from the

222 spontaneous Raman spectroscopy only (as these provided better results in the previous analyses). The average age of women diagnosed with endometrial cancer is 60 and therefore we considered this as our threshold value. Re-arranging according to age, we had the below cohorts: OC $\geq 60$ years $(n=20)$, Control $\geq 60$ years $(n=10)$, OC $<60$ years $(n=7)$, Control $<60$ years $(n=19)$. After following the same pre-processing and multivariate analysis as previous, we achieved $98 \%$ sensitivity and $90 \%$ specificity for the older group ( $\geq 60$ years) as well as $79 \%$ sensitivity and $97 \%$ specificity for the younger group (<60 years) (see SI Fig. S1).

Raman and SERS were also used to detect the early ovarian cancer cases $(n=17)$ and assess their diagnostic performance. Spontaneous Raman spectroscopy achieved 93\% sensitivity and 97\% specificity (Fig. 5A), while SERS achieved 80\% sensitivity and 94\% specificity (Fig. 5B).

\section{Discussion}

Although there has been a great effort in developing biomarkers for the early diagnosis of ovarian cancer, there is still no robust method to achieve this. This study has demonstrated the effectiveness of Raman spectroscopic methods toward the diagnosis of ovarian cancer patients, including early cases. Herein, blood plasma samples were used as a minimally invasive way of specimen collection. Blood biospectroscopy, with either infrared (IR) or Raman, has been previously evaluated in gynecological malignancy. Specifically, IR analysis of plasma and serum was applied to diagnose ovarian and endometrial cancers, providing remarkable accuracy ( $\sim 97 \%$ for ovarian and $\sim 82 \%$ for endometrial cancer) [31]; SERS analysis of plasma achieved $97 \%$ sensitivity and $92 \%$ specificity for the segregation of cervical cancer cases from normals [32]; cervical cancer and precancer were also detected with serum sample Raman spectroscopy [33]; both IR and spontaneous Raman were used to analyze blood plasma 
and serum towards the diagnosis of ovarian cancer, yielding 93\% accuracy for IR spectra and 74\% for Raman spectra of plasma [34]; Raman spectroscopy also showed promising results for ovarian cancer diagnosis in 11 patients with the disease, reaching $90 \%$ sensitivity and $100 \%$ specificity [10]; more recently, it was demonstrated that SERS was able to diagnose endometrial cancer in a pilot study using plasma and serum [35]. Some of the limitations of the above-mentioned studies include either the small number of samples or the absence of a subgroup analysis detecting early stage cases, as well as the lack of CA-125 information as a confounding factor in ovarian cancer. All of these issues have been adequately addressed in the present study. By using a satisfactory number of samples (almost 30 participants in each cohort), we managed to accurately detect both early- and late-stage ovarian cancer cases, which has not been previously shown.

In order to overcome the limitation of low signal in spontaneous Raman, SERS using AgNPs was also employed. Another advantage coming with the use of NPs is that they can be used for more specialised analysis if conjugated with targeting biomolecules, such as antibodies [36]. SERS has been shown to substantially increase the Raman signal and be beneficial for single-molecule detection; however, at the same time it presents with a number of limitations, such as lack of reproducibility and preferential metal-molecule binding, which leads to localised enhancement. This may be the reason for the decreased diagnostic accuracy when compared to spontaneous Raman. The preferential enhancement and lack of repeatability in SERS are also reflected by the increased standard deviation in the class means (Fig. 2B). Sensitivities and specificities were substantially high in both SERS (87\% and 89\%, respectively) and spontaneous Raman (94\% and 96\%, respectively), with SERS possibly being more sensitive as a biomarker extraction technique. during plasma collection. EDTA is a molecule for complexing metal ions and it has been found 
that its carboxylate groups can bind to nanoparticles surface and be responsible for the generation of new spectral bands [37]. This could potentially obscure the detection of the biological information in the derived spectra. Common anticoagulants used in plasma tubes, such as EDTA and citrate, have been previously found to interfere with SERS spectra; this was suggested to be dealt by the use of serum samples or lithium heparin as the anticoagulant [38].

Blood and its constituents are an invaluable source of information, reflecting alterations in the circulation that can be indicative of a change in health status. Recently, circulating tumour DNA (ctDNA) has attracted much attention as a blood biomarker for early and late stage malignancies, introducing an era of "liquid biopsies" [39, 40]. Also, cell-free DNA (cfDNA), reflecting both normal and ctDNA that is released after cellular necrosis and apoptosis, has been previously found significantly increased in the plasma samples of ovarian cancer patients [41]. A recent systematic review and meta-analysis of nine studies (including 462 ovarian cancer and 407 controls) concluded that cfDNA diagnosed ovarian cancer with $70 \%$ sensitivity and $90 \%$ specificity and suggested further validation and/or combination with other available biomarkers to improve the diagnostic accuracy [42]. Another study has also shown that ctDNA biomarkers could detect residual tumour, as well as predict response to treatment and survival in ovarian and endometrial cancer cases [43]. With all this in mind, it is quite possible that ctDNA fragments also contributed to the considerably high diagnostic accuracy in this spectroscopic study.

Another scope of the current study was to extract spectral biomarkers, responsible for the differentiation between the malignant and healthy individuals. Each spectral peak corresponds to chemical bonds which are present in specific biomolecules; thus, one can tentatively assign a number of disease biomarkers. To achieve this, the difference between ovarian cancer and control spectra was calculated and the ten most discriminating peaks were selected with a peak-detection algorithm; both Raman and SERS revealed five peaks in 
common and these were chosen for further statistical analysis (Fig. 3). The common peaks were correlated to proteins (Amide I and Amide III), lipids and amino acids/carbohydrates.

Surprisingly, two out of five spectral regions $\left(\sim 1657-1655 \mathrm{~cm}^{-1}\right.$ and $\left.\sim 919-916 \mathrm{~cm}^{-1}\right)$ showed inconsistency between the two spectroscopic approaches; Amide I region was decreased for ovarian cancer patients after Raman spectroscopy, while after SERS the same region was increased. Similarly, the amino acid/carbohydrate region was found decreased in ovarian cancer after Raman and increased after SERS. However, due to the fact that SERS increases significantly the signal of specific peaks, allowing thus more detailed assessment, it is possibly a more sensitive method for biomarker extraction.

More than 160 proteins have been reported to be differentially expressed in ovarian cancer, with some being upregulated, such as CA-125, CA19-9, HE4 or mesothelin, and other being downregulated, such as epidermal growth factor receptor (EGFR) and ApoA1 [44]. Amide I $\left(\sim 1650 \mathrm{~cm}^{-1}\right)$ and Amide III $\left(\sim 1300 \mathrm{~cm}^{-1}\right)$ bands represent protein molecules and are mainly associated with the $\mathrm{C}=\mathrm{O}$ stretching and $\mathrm{C}-\mathrm{N}$ stretching $/ \mathrm{N}-\mathrm{H}$ bending vibrations, respectively. The increased level of Amide I and Amide III in ovarian cancer patients after SERS, may correlate with the changes occurring due to the overexpressed proteins. The spectral bands indicative of lipids were both decreased $\left(1429 \mathrm{~cm}^{-1}\right)$ and increased $\left(1302 \mathrm{~cm}^{-1}\right)$ in the ovarian cancer group, which is also backed by previous studies showing a dysregulation of lipid metabolism in cancer [45]. For instance, some studies have shown increased lipid levels in ovarian cancer [45-47], while a limited number of studies have reported reduction [47, 48]. An alternative interpretation of the decreased lipid region $\left(1429 \mathrm{~cm}^{-1}\right)$ could be the downregulation of ApoA1 which has been previously shown to diagnose ovarian cancer in plasma and was estimated at $1484-1427 \mathrm{~cm}^{-1}$ [49]. The rise seen in the amino acids/carbohydrate region $\left(919 \mathrm{~cm}^{-1}\right)$ could potentially be attributed to ctDNA, as discussed 
previously, or correlated with increased amount of carbohydrates which is considered a risk factor for ovarian cancer $[50,51]$.

Previous spectroscopic studies investigating ovarian malignancy have not taken into account the differences between CA-125 levels, which may have led to an unrealistic segregation between patients and healthy controls. In order to investigate whether the high diagnostic accuracies achieved in our study were actually attributed to the presence of cancer or just the difference in the CA-125 levels, we also carried out a subgroup analysis to account for this. The extra analysis showed that sensitivities and specificities remained equally satisfactory which denotes that the differences found in our cohort were not attributed to CA-125 but rather to the cancerous condition. Also, after accounting for age differences, it was evident that age alone was not the reason for the high diagnostic accuracy. Even though there is a slight decrease in sensitivity and specificity (i.e., for $\geq 60$ years, specificity dropped from $96 \%$ to $90 \%$; for $<60$ years, sensitivity dropped from $94 \%$ to $79 \%$ ), the diagnostic capability remained very high.

Improved diagnostic performance for the early-stage ovarian cases was a critical objective of this study in order to allow early intervention and potentially improve patient outcomes. Again, both spectroscopic methods provided outstanding diagnostic accuracy, with Raman (sensitivity: 93\% and specificity: 97\%) being superior to SERS (sensitivity: $80 \%$ and tomography (CT), transvaginal ultrasound (TVUS) and positron emission tomography (PET) or a combination of these [52]. However, there are still a number of limitations in these methods including expense and lack of optimal sensitivity and specificity. For instance, the sensitivity and specificity of CA-125 is known to be poor, with only $50 \%$ of the patients having elevated levels of the protein at stage I and $\sim 75-90 \%$ of the cases at a later stage [4]. CA-125 level can be used more reliably to monitor treatment as levels of CA-125 decrease when a treatment is 
efficient. However, it is not useful for screening as CA-125 level can be elevated in other conditions, such as endometriosis, breast or lung malignancies, and also not every woman with ovarian cancer has elevated CA-125; CT is expensive and has high false-positive rates which prevent its use in screening [1]. Even though TVUS is preferred than other imaging techniques in terms of speed and sensitivity, there is yet no convincing evidence that it detects early ovarian cancer without causing overtreatment of non-malignant cases [2]. TVUS can indeed show a mass in the ovary but it cannot distinguish whether the mass is benign or malignant. Therefore, other blood biomarkers (CA-125) are used together with ultrasound to identify ovarian tumour at high risk of malignancy. Previous large cohort studies have evaluated the sensitivity and specificity of multimodal screening (MMS) (i.e., annual testing of CA-125 with ultrasound scan as a second line test) and ultrasound screening (USS) (i.e., ultrasound alone); their results showed that the MMS gave slightly higher sensitivity [5, 53]. Specifically, the overall sensitivity for detection of ovarian cancers, diagnosed within a year of a screening, was $84 \%$ in the MMS group and $73 \%$ in the USS group [5]. However, the positive predictive value for USS was estimated at around $\sim 5 \%$, which indicates a quite high false-positive rate [53]. In an effort to improve the diagnostic accuracy many groups have also combined different biomarkers, which however increase the cost and time requirement [1, 44]. By using spectroscopic techniques these drawbacks seem to be eliminated as they provide a simpler, cost-effective, multi-marker assay, thus securing robustness. The diagnostic accuracy shown in this study is even better than the currently used tests.

In conclusion, the efficacy of Raman spectroscopic methods (i.e., spontaneous Raman and SERS) in detecting ovarian cancer, including early-stage patients, has been demonstrated. Continuous efforts are being made to improve clinical diagnosis and monitoring of disease in ovarian cancer. Our findings suggest improved diagnostic accuracy compared to traditional biomarkers. Specific biomolecules were also found responsible for the segregation between the 
cancer and healthy cases and could be used as spectral biomarkers. Future spectroscopic studies

370

371

372

373

374

375

376

377

378

379

380

381

382

383

384

385

386

387

388

389

390

391

392

393

394

395

396

397

398

399

400

401

should focus on the validation of these results in larger datasets and across different scientific groups and laboratories; this would open a new road in ovarian cancer research and potentially allow the implementation of blood spectroscopy in clinical practice as a promising diagnostic tool.

\section{Acknowledgements}

MP acknowledges Rosemere Cancer Foundation for funding. Special thanks to Emma Callery and Debra Beesley for conducting the serum CA-125 tests.

\section{Availability of data and materials}

All raw and pre-processed spectra will be available at the publicly accessible data repository Figshare.

\section{Disclosure/Conflict of Interest}

The authors declare no conflicts of interest

\section{Authorship}

FLM and PLMH conceived the study; MP designed the study, conducted the spectroscopic, multivariate/statistical analysis and wrote the manuscript; KA, HFS, NW, PK, AR and PLMH collected and provided the samples. All authors provided constructive feedback during manuscript preparation. All authors have approved the final version.

\section{References}

[1] Z. Yurkovetsky, S. Skates, A. Lomakin, B. Nolen, T. Pulsipher, F. Modugno, J. Marks, A. Godwin, E. Gorelik, I. Jacobs, Development of a multimarker assay for early detection of ovarian cancer, J Clin Oncol 28(13) (2010) 2159-2166.

[2] G.C. Jayson, E.C. Kohn, H.C. Kitchener, J.A. Ledermann, Ovarian cancer, Lancet 384(9951) (2014) 1376-1388.

[3] C.H. Holschneider, J.S. Berek, Ovarian cancer: epidemiology, biology, and prognostic factors, Semin Surg Oncol, Wiley Online Library, 2000, pp. 3-10.

[4] E. Moss, J. Hollingworth, T. Reynolds, The role of CA125 in clinical practice, J. Clin. Pathol. 58(3) (2005) 308-312.

[5] I.J. Jacobs, U. Menon, A. Ryan, A. Gentry-Maharaj, M. Burnell, J.K. Kalsi, N.N. Amso, S. Apostolidou, E. Benjamin, D. Cruickshank, Ovarian cancer screening and mortality in the UK Collaborative Trial of Ovarian Cancer Screening (UKCTOCS): a randomised controlled trial, Lancet 387(10022) (2016) 945-956. 

Enomoto, Biomarkers for screening, diagnosis, and monitoring of ovarian cancer, Cancer Epidemiol Biomarkers Prevent 21(11) (2012) 1902-1912. [7] F. Su, J. Lang, A. Kumar, C. Ng, B. Hsieh, M.A. Suchard, S.T. Reddy, R. Farias-Eisner, Validation of candidate serum ovarian cancer biomarkers for early detection, Biomark Insights 2 (2007) 369. detection of ovarian cancer: from traditional methods to proteomics. Can we really do better than serum CA-125?, Am J Obstet Gynecol 199(3) (2008) 215-223.

[9] I. Visintin, Z. Feng, G. Longton, D.C. Ward, A.B. Alvero, Y. Lai, J. Tenthorey, A. Leiser, R. FloresSaaib, H. Yu, Diagnostic markers for early detection of ovarian cancer, Clin Cancer Res 14(4) (2008) 1065-1072.

[10] I. Ullah, I. Ahmad, H. Nisar, S. Khan, R. Ullah, R. Rashid, H. Mahmood, Computer assisted optical screening of human ovarian cancer using Raman spectroscopy, Photodiagnosis Photodyn Ther 15 (2016) 94-99.

[11] I. Pence, A. Mahadevan-Jansen, Clinical instrumentation and applications of Raman spectroscopy, Chem Soc Rev 45(7) (2016) 1958-1979.

[12] I.P. Santos, E.M. Barroso, T.C. Bakker Schut, P.J. Caspers, C.G.F. van Lanschot, D.H. Choi, M.F. van der Kamp, R.W.H. Smits, R. van Doorn, R.M. Verdijk, V. Noordhoek Hegt, J.H. von der Thusen, C.H.M. van Deurzen, L.B. Koppert, G. van Leenders, P.C. Ewing-Graham, H.C. van Doorn, C.M.F. Dirven, M.B. Busstra, J. Hardillo, A. Sewnaik, I. Ten Hove, H. Mast, D.A. Monserez, C. Meeuwis, T. Nijsten, E.B. Wolvius, R.J. Baatenburg de Jong, G.J. Puppels, S. Koljenovic, Raman spectroscopy for cancer detection and cancer surgery guidance: translation to the clinics, Analyst 142 (2017) 3025-47. [13] F.L. Cals, T.C. Bakker Schut, J.A. Hardillo, R.J. Baatenburg de Jong, S. Koljenovic, G.J. Puppels, Investigation of the potential of Raman spectroscopy for oral cancer detection in surgical margins, Lab Invest 95(10) (2015) 1186-96.

[14] M.D. Keller, E. Vargis, N. de Matos Granja, R.H. Wilson, M.A. Mycek, M.C. Kelley, A. MahadevanJansen, Development of a spatially offset Raman spectroscopy probe for breast tumor surgical margin evaluation, J Biomed Opt 16(7) (2011) 077006.

[15] K. Kong, C. Kendall, N. Stone, I. Notingher, Raman spectroscopy for medical diagnostics - From in-vitro biofluid assays to in-vivo cancer detection, Adv Drug Del Rev 89 (2015) 121-134.

[16] M. Jermyn, K. Mok, J. Mercier, J. Desroches, J. Pichette, K. Saint-Arnaud, L. Bernstein, M.C. Guiot, K. Petrecca, F. Leblond, Intraoperative brain cancer detection with Raman spectroscopy in humans, Sci Transl Med 7(274) (2015) 274 ra19.

[17] F.M. Lyng, D. Traynor, I.R. Ramos, F. Bonnier, H.J. Byrne, Raman spectroscopy for screening and diagnosis of cervical cancer, Anal Bioanal Chem 407(27) (2015) 8279-89.

[18] M. Paraskevaidi, C.L. Morais, D.E. Halliwell, D.M. Mann, D. Allsop, P.L. Martin-Hirsch, F.L. Martin, Raman spectroscopy to diagnose Alzheimer's disease and dementia with Lewy bodies in blood, ACS Chem Neurosci (2018).

[19] M. Paraskevaidi, C. de Morais, K.M.G. de Lima, K. Ashton, H.F. Stringfellow, P.L. Martin-Hirsch, F.L. Martin, Potential of mid-infrared spectroscopy as a non-invasive diagnostic test in urine for endometrial or ovarian cancer, Analyst (2018).

[20] C.M. Krishna, G. Sockalingum, L. Venteo, R.A. Bhat, P. Kushtagi, M. Pluot, M. Manfait, Evaluation of the suitability of ex vivo handled ovarian tissues for optical diagnosis by Raman microspectroscopy, Biopolymers 79(5) (2005) 269-276.

[21] R. Mehrotra, G. Tyagi, D.K. Jangir, R. Dawar, N. Gupta, Analysis of ovarian tumor pathology by Fourier Transform Infrared Spectroscopy, J Ovarian Res 3(1) (2010) 27.

[22] G. Theophilou, K.M.G. Lima, P.L. Martin-Hirsch, H.F. Stringfellow, F.L. Martin, ATR-FTIR spectroscopy coupled with chemometric analysis discriminates normal, borderline and malignant ovarian tissue: classifying subtypes of human cancer, Analyst 141(2) (2016) 585-594. 

of biofluids for disease screening or diagnosis: translation from the laboratory to a clinical setting, J Biophotonics 7(3-4) (2014) 153-165. [24] M. Paraskevaidi, C.L. Morais, K.M. Lima, J.S. Snowden, J.A. Saxon, A.M. Richardson, M. Jones, spectrochemical analysis of blood, Proc Natl Acad Sci USA (2017) 201701517. [25] N. MacRitchie, G. Grassia, J. Noonan, P. Garside, D. Graham, P. Maffia, Molecular imaging of atherosclerosis: spotlight on Raman spectroscopy and surface-enhanced Raman scattering, Heart (2017) heartjnl-2017-311447.

[26] B. Fazio, C. D'Andrea, A. Foti, E. Messina, A. Irrera, M.G. Donato, V. Villari, N. Micali, O.M. Maragò, P.G. Gucciardi, SERS detection of Biomolecules at Physiological pH via aggregation of Gold Nanorods mediated by Optical Forces and Plasmonic Heating, Sci Rep 6 (2016) 26952. [27] H.J. Butler, L. Ashton, B. Bird, G. Cinque, K. Curtis, J. Dorney, K. Esmonde-White, N.J. Fullwood, B. Gardner, P.L. Martin-Hirsch, M.J. Walsh, M.R. McAinsh, N. Stone, F.L. Martin, Using Raman spectroscopy to characterize biological materials, Nat Protoc 11(4) (2016) 664-687. [28] M.J. Baker, J. Trevisan, P. Bassan, R. Bhargava, H.J. Butler, K.M. Dorling, P.R. Fielden, S.W. Fogarty, N.J. Fullwood, K.A. Heys, C. Hughes, P. Lasch, P.L. Martin-Hirsch, B. Obinaju, G.D. Sockalingum, J. Sulé-Suso, R.J. Strong, M.J. Walsh, B.R. Wood, P. Gardner, F.L. Martin, Using Fourier transform IR spectroscopy to analyze biological materials, Nat Protoc 9(8) (2014) 1771-1791. [29] C.-W. Hsu, C.-C. Chang, C.-J. Lin, A practical guide to support vector classification, (2003), (https://www.researchgate.net/profile/Chenghai Yang/publication/272039161 Evaluating unsuper vised and supervised image classification methods for mapping cotton root rot/links/55f2c574 08ae0960a3897985/Evaluating-unsupervised-and-supervised-image-classification-methods-formapping-cotton-root-rot.pdf).

[30] J. Trevisan, P.P. Angelov, P.L. Carmichael, A.D. Scott, F.L. Martin, Extracting biological information with computational analysis of Fourier-transform infrared (FTIR) biospectroscopy datasets: current practices to future perspectives, Analyst 137(14) (2012) 3202-15.

[31] K. Gajjar, J. Trevisan, G. Owens, P.J. Keating, N.J. Wood, H.F. Stringfellow, P.L. Martin-Hirsch, F.L. Martin, Fourier-transform infrared spectroscopy coupled with a classification machine for the analysis of blood plasma or serum: a novel diagnostic approach for ovarian cancer, Analyst 138(14) (2013) 3917-26.

[32] S. Feng, D. Lin, J. Lin, B. Li, Z. Huang, G. Chen, W. Zhang, L. Wang, J. Pan, R. Chen, Blood plasma surface-enhanced Raman spectroscopy for non-invasive optical detection of cervical cancer, Analyst 138(14) (2013) 3967-3974.

[33] J.L. González-Solís, J.C. Martínez-Espinosa, L.A. Torres-González, A. Aguilar-Lemarroy, L.F. JaveSuárez, P. Palomares-Anda, Cervical cancer detection based on serum sample Raman spectroscopy, Lasers Med Sci 29(3) (2014) 979-985.

[34] G.L. Owens, K. Gajjar, J. Trevisan, S.W. Fogarty, S.E. Taylor, B. Da Gama-Rose, P.L. Martin-Hirsch, F.L. Martin, Vibrational biospectroscopy coupled with multivariate analysis extracts potentially diagnostic features in blood plasma/serum of ovarian cancer patients, J Biophotonics 7(3-4) (2014) 200-9.

[35] H.J. Butler, S.W. Fogarty, J.G. Kerns, P.L. Martin-Hirsch, N.J. Fullwood, F.L. Martin, Gold nanoparticles as a substrate in bio-analytical near-infrared surface-enhanced Raman spectroscopy, Analyst 140(9) (2015) 3090-3097.

[36] M. Arruebo, M. Valladares, Á. González-Fernández, Antibody-conjugated nanoparticles for biomedical applications, J Nanomater 2009 (2009) 37.

[37] S. Mondal, S. Verma, Catalytic and SERS Activities of Tryptophan-EDTA Capped Silver Nanoparticles, Z Anorg Allg Chem 640(6) (2014) 1095-1101.

[38] A. Bonifacio, S. Dalla Marta, R. Spizzo, S. Cervo, A. Steffan, A. Colombatti, V. Sergo, Surfaceenhanced Raman spectroscopy of blood plasma and serum using Ag and Au nanoparticles: a systematic study, Anal Bioanal Chem 406(9-10) (2014) 2355-2365. 
[39] C. Bettegowda, M. Sausen, R.J. Leary, I. Kinde, Y. Wang, N. Agrawal, B.R. Bartlett, H. Wang, B. Luber, R.M. Alani, Detection of circulating tumor DNA in early-and late-stage human malignancies, Sci Transl Med 6(224) (2014) 224ra24-224ra24.

[40] L.A. Diaz Jr, A. Bardelli, Liquid biopsies: genotyping circulating tumor DNA, J Clin Oncol 32(6) (2014) 579-586.

[41] A.A. Kamat, M. Baldwin, D. Urbauer, D. Dang, L.Y. Han, A. Godwin, B.Y. Karlan, J.L. Simpson, D.M. Gershenson, R.L. Coleman, Plasma cell-free DNA in ovarian cancer, Cancer 116(8) (2010) 19181925.

[42] Q. Zhou, W. Li, B. Leng, W. Zheng, Z. He, M. Zuo, A. Chen, Circulating cell free DNA as the diagnostic marker for ovarian cancer: A systematic review and meta-analysis, PloS one 11(6) (2016) e0155495.

[43] E. Pereira, O. Camacho-Vanegas, S. Anand, R. Sebra, S.C. Camacho, L. Garnar-Wortzel, N. Nair, E. Moshier, M. Wooten, A. Uzilov, Personalized circulating tumor DNA biomarkers dynamically predict treatment response and survival in gynecologic cancers, PLoS One 10(12) (2015) e0145754.

[44] B.M. Nolen, A.E. Lokshin, Protein biomarkers of ovarian cancer: the forest and the trees, Future Oncol 8(1) (2012) 55-71.

[45] C.R. Santos, A. Schulze, Lipid metabolism in cancer, FEBS J. 279(15) (2012) 2610-2623.

[46] I. Delimaris, E. Faviou, G. Antonakos, E. Stathopoulou, A. Zachari, A. Dionyssiou-Asteriou, Oxidized LDL, serum oxidizability and serum lipid levels in patients with breast or ovarian cancer, Clin Biochem 40(15) (2007) 1129-1134.

[47] M. Tania, M. Khan, Y. Song, Association of lipid metabolism with ovarian cancer, Curr Oncol 17(5) (2010) 6.

[48] Q. Hu, M. Wang, M.S. Cho, C. Wang, A.M. Nick, P. Thiagarajan, F.M. Aung, X. Han, A.K. Sood, V. Afshar-Kharghan, Lipid profile of platelets and platelet-derived microparticles in ovarian cancer, BBA clinical 6 (2016) 76-81.

[49] G. Deleris, C. Petibois, Applications of FT-IR spectrometry to plasma contents analysis and monitoring, Vib Spectrosc 32(1) (2003) 129-136.

[50] L. Augustin, J. Polesel, C. Bosetti, C. Kendall, C. La Vecchia, M. Parpinel, E. Conti, M. Montella, S. Franceschi, D. Jenkins, Dietary glycemic index, glycemic load and ovarian cancer risk: a case-control study in Italy, Ann Oncol 14(1) (2003) 78-84.

[51] B. Qin, P.G. Moorman, A.J. Alberg, J.S. Barnholtz-Sloan, M. Bondy, M.L. Cote, E. Funkhouser, E.S. Peters, A.G. Schwartz, P. Terry, Dietary carbohydrate intake, glycaemic load, glycaemic index and ovarian cancer risk in African-American women, Br J Nutr 115(4) (2016) 694-702.

[52] J.V. Jokerst, A.J. Cole, D. Van de Sompel, S.S. Gambhir, Gold nanorods for ovarian cancer detection with photoacoustic imaging and resection guidance via Raman imaging in living mice, ACS Nano 6(11) (2012) 10366-10377.

[53] U. Menon, A. Gentry-Maharaj, R. Hallett, A. Ryan, M. Burnell, A. Sharma, S. Lewis, S. Davies, S. Philpott, A. Lopes, Sensitivity and specificity of multimodal and ultrasound screening for ovarian cancer, and stage distribution of detected cancers: results of the prevalence screen of the UK Collaborative Trial of Ovarian Cancer Screening (UKCTOCS), Lancet Oncol 10(4) (2009) 327-340. [54] Z. Movasaghi, S. Rehman, I.U. Rehman, Raman spectroscopy of biological tissues, Appl Spectrosc Rev 42(5) (2007) 493-541.

[55] S. Li, L. Li, Q. Zeng, Y. Zhang, Z. Guo, Z. Liu, M. Jin, C. Su, L. Lin, J. Xu, S. Liu, Characterization and noninvasive diagnosis of bladder cancer with serum surface enhanced Raman spectroscopy and genetic algorithms, Sci Rep 5 (2015) 9582.

[56] D. Lin, S. Feng, J. Pan, Y. Chen, J. Lin, G. Chen, S. Xie, H. Zeng, R. Chen, Colorectal cancer detection by gold nanoparticle based surface-enhanced Raman spectroscopy of blood serum and statistical analysis, Opt Express 19(14) (2011) 13565-13577.

[57] S. Feng, R. Chen, J. Lin, J. Pan, G. Chen, Y. Li, M. Cheng, Z. Huang, J. Chen, H. Zeng, Nasopharyngeal cancer detection based on blood plasma surface-enhanced Raman spectroscopy and multivariate analysis, Biosens Bioelectron 25(11) (2010) 2414-2419. 
553

554

555

556

557

558

559

560

561

562

563

564

565

566

567

568

569

570

571

572

573

574

575

576

577

578

579

580

581

582

583
[58] J.L. Pichardo-Molina, C. Frausto-Reyes, O. Barbosa-García, R. Huerta-Franco, J.L. GonzálezTrujillo, C.A. Ramírez-Alvarado, G. Gutiérrez-Juárez, C. Medina-Gutiérrez, Raman spectroscopy and multivariate analysis of serum samples from breast cancer patients, Lasers Med Sci 22(4) (2007) 229236.

.

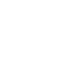

2

363


584 Figure Legends

A

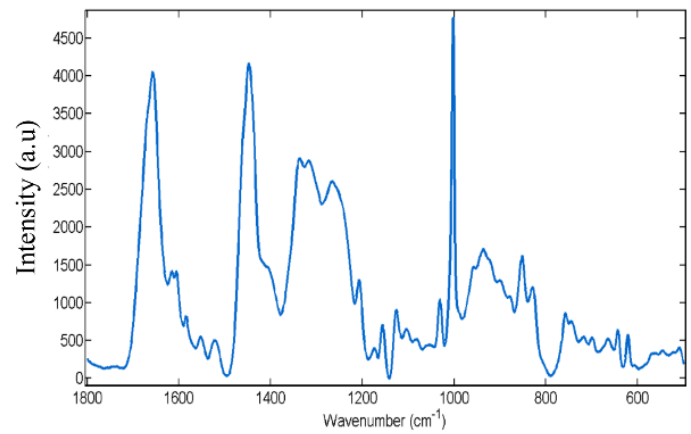

B

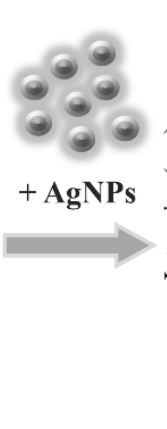

SERS

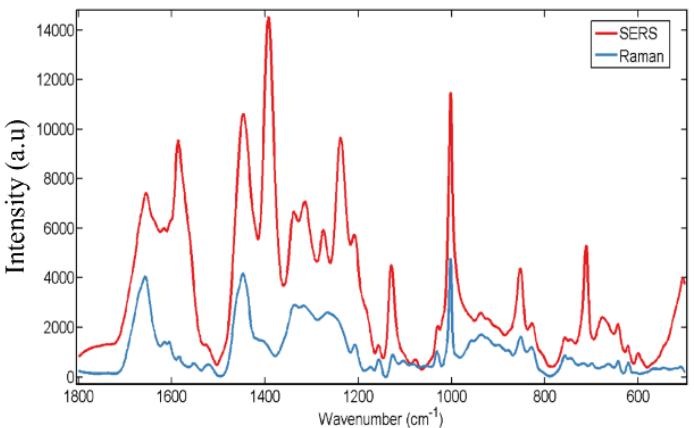

585

586 Figure 1: Enhancement effect of SERS after the addition of silver nanoparticles (AgNPs) in 587 blood samples.

588

589

590 
A

Raman

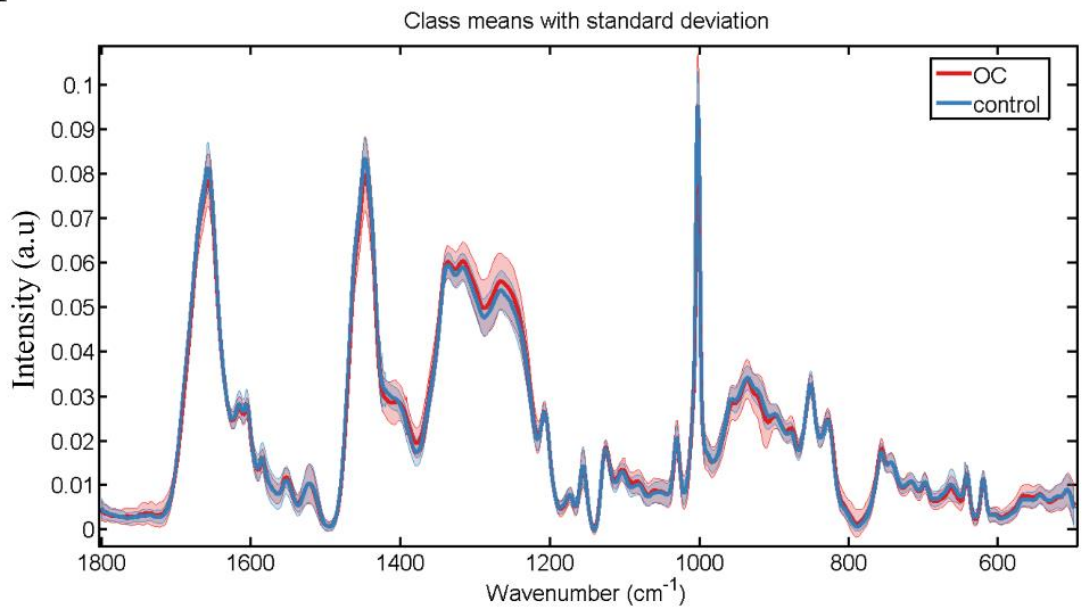

Sensitivity: $94 \%$

Specificity: $96 \%$

B

\section{SERS}

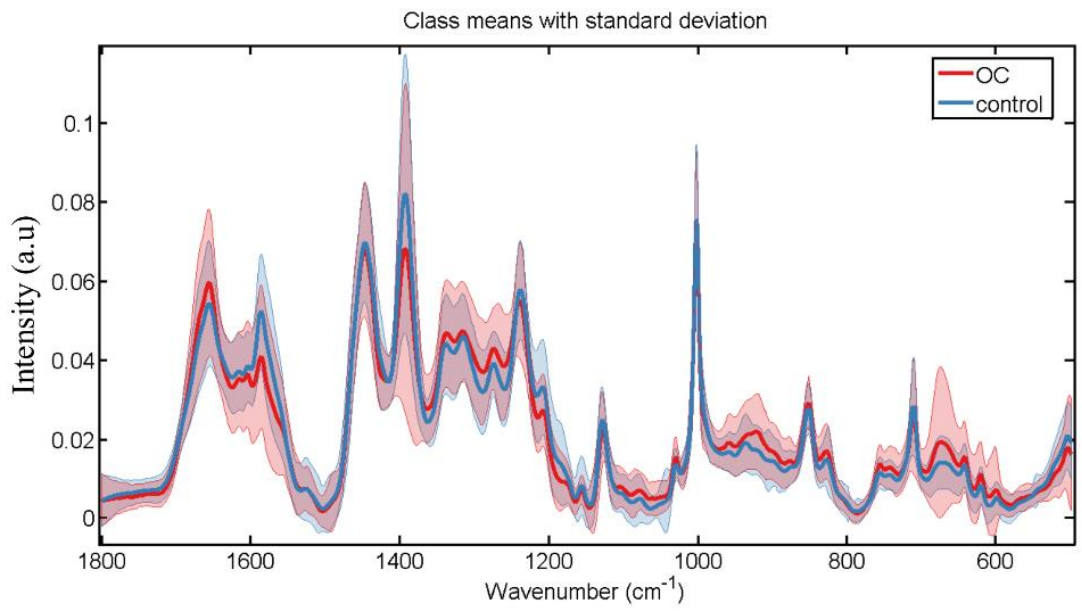

Sensitivity: $\mathbf{8 7 \%}$

Specificity: $\mathbf{8 9 \%}$

591

592 Figure 2: Diagnostic segregation of ovarian cancer (OC) with (A) Raman spectroscopy and 593 (B) SERS. 

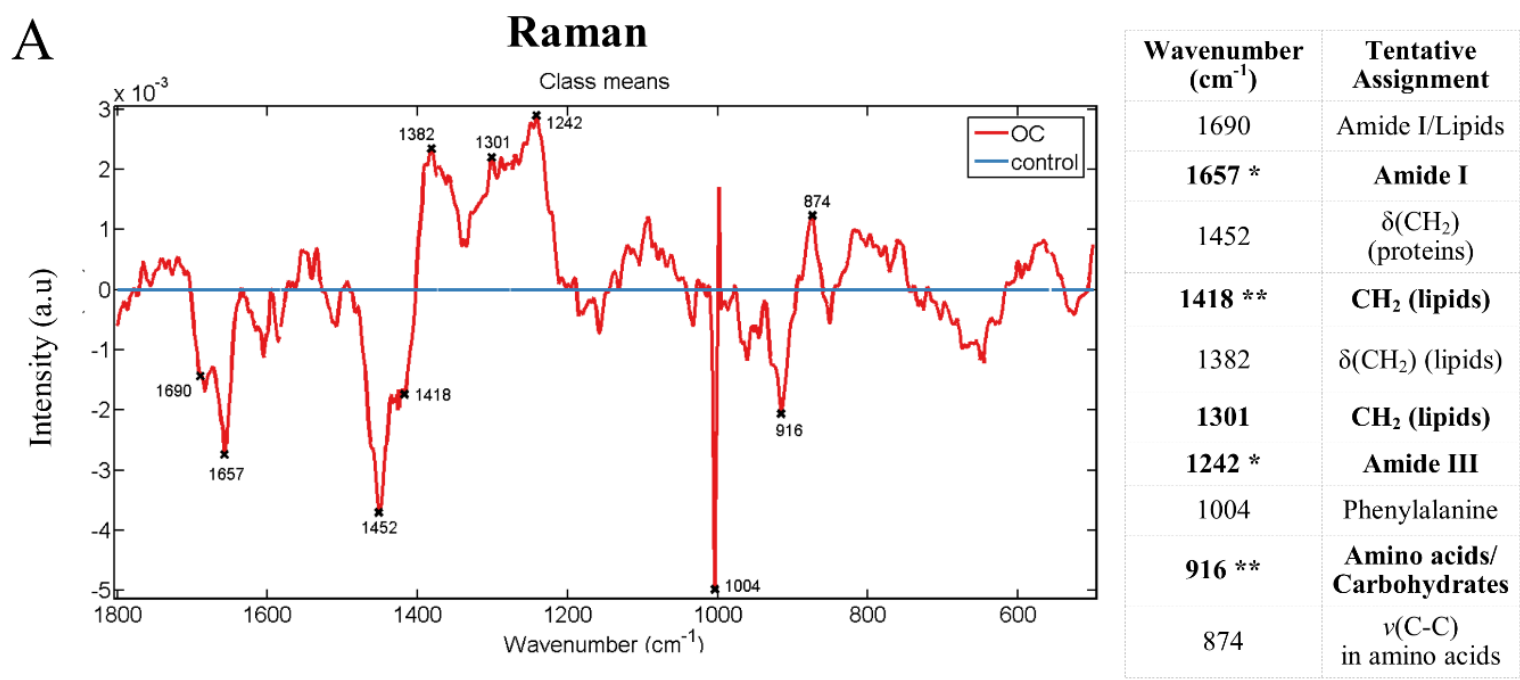

$\mathrm{B}$
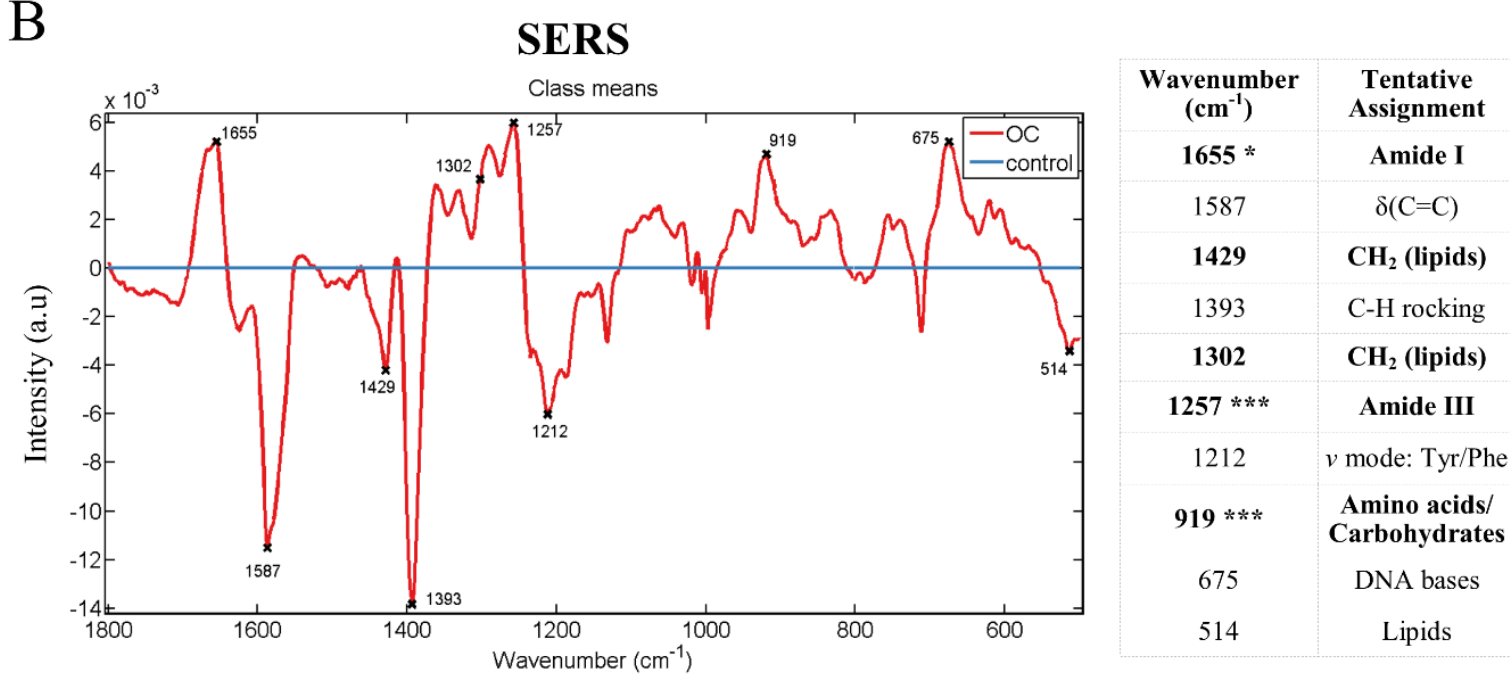

595 Figure 3: Differentiating spectral peaks after (A) Raman spectroscopy and (B) SERS. The 596 tables show the peak positions and tentative assignments of major vibrational bands [54-58]; 597 peaks shown with bold were detected with both Raman and SERS and may be used as more 598 reliable diagnostic biomarkers. Abbreviations: OC: ovarian cancer; $v$ : stretching mode; $\delta$ : 599 bending mode. 


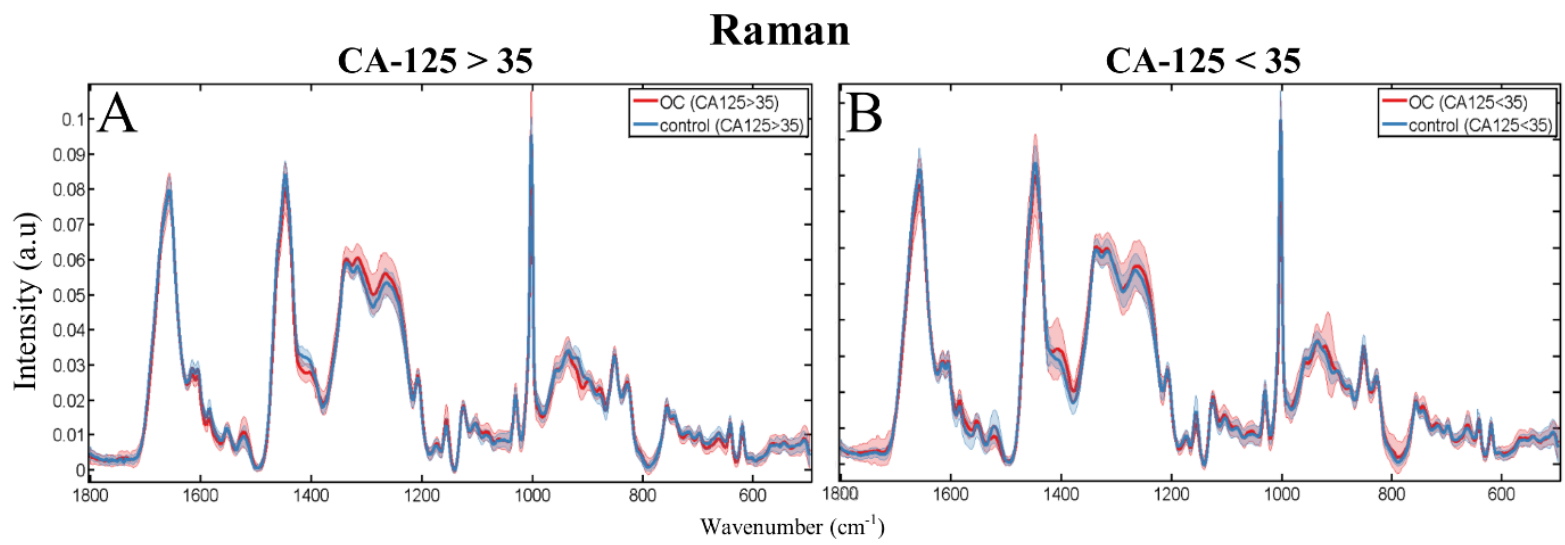

Sensitivity: 99\%

Sensitivity: $\mathbf{7 8 \%}$

Specificity: $\mathbf{8 5 \%}$

Specificity: $\mathbf{9 9 \%}$

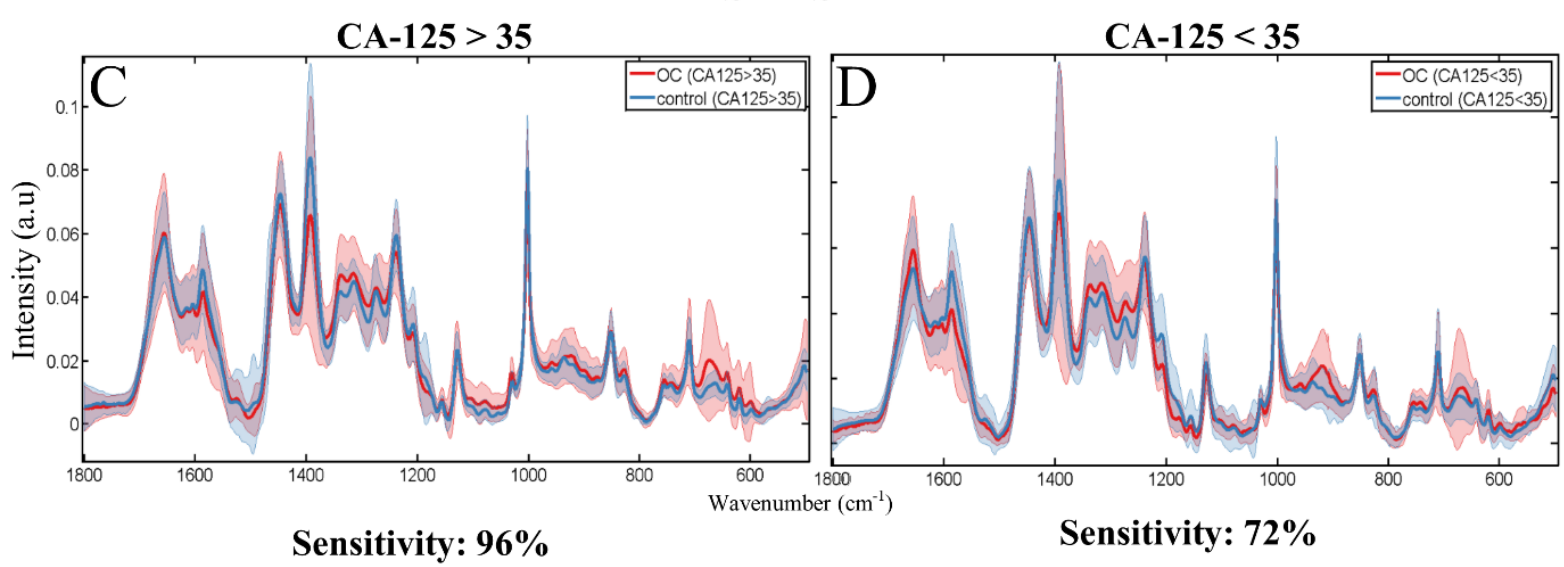

Specificity: $74 \%$

Specificity: $\mathbf{9 7 \%}$

600

601

602

603

604

605

Figure 4: Diagnostic segregation between ovarian cancer (OC) patients and healthy controls according to their CA-125 levels. Sensitivity and specificity are provided for (A) individuals with CA-125>35 u/ml after Raman analysis, (B) individuals with CA-125<35 u/ml after Raman, (C) individuals with CA-125>35 u/ml after SERS and (D) individuals with CA-125<35 $\mathrm{u} / \mathrm{ml}$ after SERS. 
A

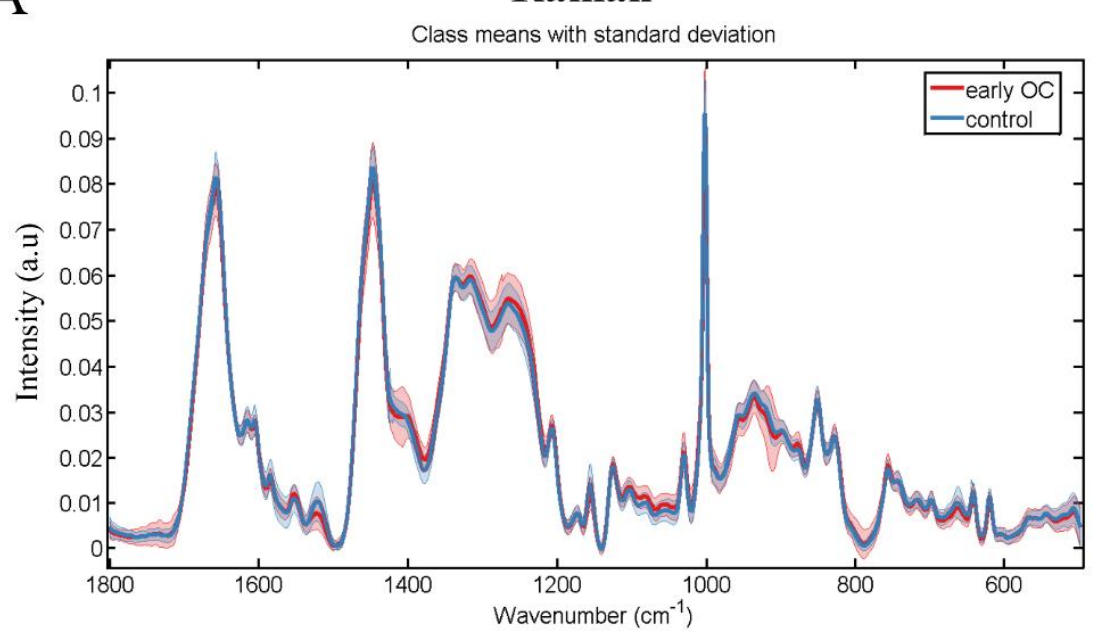

B

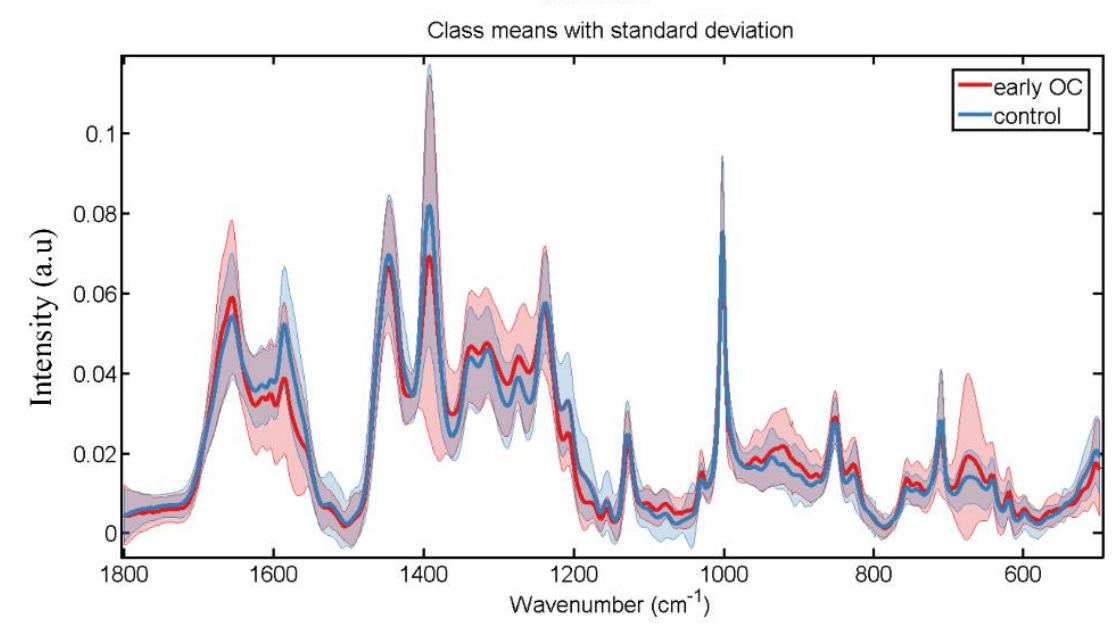

Sensitivity: $93 \%$

Specificity: 97\%

Sensitivity: $\mathbf{8 0 \%}$

Specificity: 94\%

607 Figure 5: Diagnosis of early ovarian cancer (OC) after (A) Raman spectroscopy and (B) SERS. 